\title{
Impact of Seed-borne Colletotrichum lindemuthianum on French Bean Seed Quality
}

\author{
S. RAVI, SABITHA DORAISWAMY, V. VALLUVAPARIDASAN \\ and C. JEYALAKSHMI \\ Department of Plant Pathology, Centre for Plant Protection Studies, \\ Tamil Nadu Agricultural University, Coimbatore - 641 003, Tamilnadu, India
}

\begin{abstract}
French bean seed samples were collected from different parts of Tamil Nadu including both tropical and temperate region including four cultivars viz., Arkakomal, Premier, Rajmash DPU 88-4 and Watex. The variety Watex was found to maximum per cent seed infection of $C$. lindemuthianum with more discolorations. Among the various techniques adopted for detection, agar plate method was found to be best and yielded maximum per cent seed infection. Direct correlation was obtained between seed discoloration and per cent seed infection. The pathogen was found to be present mostly in seed coat and cotyledon and rarely in embryonic axis. The artificial inoculation of 45-day-old plants resulted in maximum disease incidence, resultant seed infection and minimum seed yield /plant and 100 grain weight.
\end{abstract}

Key words: Colletotrichum lindemuthianum, French bean

French bean (Phaseolus vulgaris L.) is a highly priced proteinaceous vegetable crop of India being severely affected by anthracnose incited by Colletotrichum lindemuthianum. As an important seed-borne pathogen, it inflicts considerable damage to the crop, reduces the seed quality and yield. Goulart (1988) and Tylkowska and Dorna (1990) described anthracnose incidence and seed transmission of the pathogen. The severely affected pods shows characteristic brown lesions with white centre, surrounded by a pale brown to dark brown area of various size (Ansleme and Champion, 1981) and the pathogen survive mostly in cotyledons (Zaumeyer and Thomas, 1957). Keeping in mind, an attempt was made to standardize the detection of seed borne $C$. lindemuthianum, its site of infection, impact on seed quality and seedling vigour and susceptibility of plant age on disease incidence and seed transmission in four popularly cultivated french bean cultivars of Tamilnadu.

\section{Materials and Methods}

\section{(i) Collection of seed samples}

French bean (each $1 \mathrm{~kg}$ ) variety viz., Arkakomal (Hosur, Madurai, Melchengam, Ooty); Premier (Gudiatham, Hosur, Kodaikonal and Tirupathur); Rajmash DPU-88-4 (Kelamangalam, Nanjanadu, Ooty and Yercaud) and Watex (Hosur, Kelamangalam, Nanjanadu and Ooty) harvested during the previous season were collected and used for the detection and isolation of Colletotrichum lindemuthianum. 
(ii) Detection and isolation of seed-borne C. lindemuthianum

Detection of seed-borne $C$. lindemuthianum was made as per standard blotter method (ISTA, 1985), 0.1\% 2,4-D blotter method (Neergaard Paul and Saab Adib, 1962), deep freeze method (Limonard, 1966) and agar plate method (Mew and Misra, 1974). The fungus was isolated from the naturally infected french bean seeds by transferring the acervulus into Potato Dextrose Agar (PDA) under aseptic condition, with the help of steriobinocular microscope. The pathogen was further purified by single spore isolation and maintained on PDA slants.

(iii) Impact of seed-borne C. lindemuthianum on germination

This experiment was conducted by following the procedure outlined by ISTA (1985) in sand medium. One hundred seeds of each seed sample were sown in a germination tray contains sterilized moistened sand and incubated in a germination room, maintained at $25 \pm 2{ }^{\circ} \mathrm{C}$ and $90 \pm 3$ per cent $\mathrm{RH}$. Four replications were maintained for each seed sample. The number of normal seedlings, abnormal seedlings and dead seeds in each replications were counted ninth day after sowing and per cent of germination, abnormal seedling and dead seed were calculated and the results were tabulated.

(iv) Assessment of $\mathrm{C}$. lindemuthianum infection in discoloured seeds

Infected seed lots of different variety viz., Arkakomal, Premier, Rajmash DPU88-4 and Watex showing varying degrees of discolouration were used in this study. Each seed sample was homogenized as per sampling technique (ISTA, 1985); divided into four working samples and treated as four replications. In each replication, 100 seeds were counted separately and the per cent of discoloured seed was worked out. Then the discoloured seeds were plated on blotter paper. The percentage of $C$. lindemuthianum associated with discoloured seeds was recorded.

(v) Effect of $\mathrm{C}$. lindemuthianum seed infection on seed size and weight

In order to find out the effect of seed infection on quality of french bean seeds, a pot culture experiment was conducted. Thirty-day-old french bean varieties viz., Arkakomal, Premier, Rajmash DPU 88-4 and Watex raised in earthen pots were sprayed with $C$. lindemuthianum spore suspension $\left(10^{6}\right.$ spore $\left./ \mathrm{ml}\right)$ along with Tween 20 . Suitable control was also maintained. Finally, the seeds were harvested separately, processed and dried to bring down the moisture content to less than 9 per cent. The seeds harvested from these plants were used for assessing $C$. lindemuthianum infection as mentioned earlier.

(iv-a) On seed weight

In order to find out the effect of $C$. lindemuthianum on seed infection, 100 seed weight was taken individually for both infected and healthy seeds in all the four varieties under test and five replications were maintained in each. The percentage of seed infection was also recorded by agar plate method. 
(iv-b) On seed size

The length and breadth of both infected and healthy seeds in the four varieties were measured with the help of a screw gauge. Five replications were maintained in each variety. One hundred seeds formed one replication.

\section{(vi) Site of infection}

To find out the location of $C$. lindemuthianum infection in infected french bean seeds, seed component plating method (Kunwar et al., 1985) was followed.

One hundred and twenty-five asymptotic and symptomatic seeds were soaked separately in sterilized double distilled water with one seed per well (Template) for $6 \mathrm{~h}$. Soaked seeds were then dissected aseptically into three components viz., the seed coat, cotyledon and embryonic axis. Each component was surface sterilized with 0.1 per cent Sodium hypochlorite for five minutes and then washed in two changes of sterile doubledistilled water. The components of individual seeds were plated on Petri plates containing three layers of moistened filter paper and incubated as per ISTA (1985). Twenty-five seeds formed one replication and five replications were maintained in each variety.

(vii) Effect of plant age on seed infection and seed transmission of $\mathrm{C}$. lindemuthianum

Highly susceptible french bean variety Watex was used in this experiment. The plants were maintained in pots with twenty plants per replication. Spore suspension of the pathogen $\left(10^{6}\right.$ spore/ml concentration) was sprayed on 15-, 30-, 45- and 60-day-old plants. Four replications were maintained. The per cent disease incidence, per cent pod infection were recorded 15 days after last spray. The seeds were harvested and per cent seed infection in the resultant seeds, seed yield/plant and 100 seed weight were recorded.

\section{Results and Discussion}

Sixteen french bean samples consisting of four varieties were collected from different parts of Tamil Nadu and were subjected for seed health testing to assess the per cent occurrence of $C$. lindemuthianum seed infection. Among the four techniques followed, agar plate method and blotter technique gave better results when compared to 2,4-D and deep freeze method (Table 1). In conformity with the above findings, Tylkowska and Dorna (1990) reported that potato dextrose agar medium with streptomycin gave better result for the detection of $C$. lindemuthianum compared to roll towel method. The maximum seed infection was noticed in seed samples collected from temperate and sub tropical regions (Ooty, Nanjanadu, Kodaikanal, Yercaud and Hosur) compared to tropical regions (Gudiatham, Madurai, Melchengam, Tirupathur and Kelamangaklam). Similar findings on high rate of anthracnose incidence and seed transmission of C. lindemuthianum in french bean was also reported by Arya et al. (1986) and Davis (1988). Out of four varieties tested, the variety Watex was found to show maximum seed infection compared to others. 


\section{Table 1}

Occurrence of seed borne $C$. lindemuthianum in french bean culture

\begin{tabular}{|c|c|c|c|c|c|}
\hline \multirow[b]{2}{*}{ Variety } & \multirow{2}{*}{$\begin{array}{l}\text { Place of } \\
\text { collection }\end{array}$} & \multicolumn{4}{|c|}{ Seed infection $(\%)^{*}$} \\
\hline & & $\begin{array}{c}\text { Standard } \\
\text { blotter }\end{array}$ & $\begin{array}{c}1 \% \\
2,4-\mathrm{D}\end{array}$ & $\begin{array}{l}\text { Deep } \\
\text { freeze }\end{array}$ & $\begin{array}{l}\text { Agar } \\
\text { plate }\end{array}$ \\
\hline \multirow[t]{4}{*}{ Arkakomal } & Hosur & $\begin{array}{c}70.4^{\mathrm{h}} \\
(57.21)\end{array}$ & $\begin{array}{c}28.8^{\mathrm{e}} \\
(32.31)\end{array}$ & $\begin{array}{c}14.4^{\mathrm{e}} \\
(22.28)\end{array}$ & $\begin{array}{c}76.0^{\mathrm{f}} \\
(61.20)\end{array}$ \\
\hline & Madurai & $\begin{array}{c}24.8^{\mathrm{d}} \\
(29.68)\end{array}$ & $\begin{array}{c}9.6^{c} \\
(17.65)\end{array}$ & $\begin{array}{c}5.6^{\mathrm{c}} \\
(13.69)\end{array}$ & $\begin{array}{c}40.0^{c} \\
(39.96)\end{array}$ \\
\hline & Melchengam & $\begin{array}{c}21.6^{c} \\
(27.15)\end{array}$ & $\begin{array}{c}4.0^{\mathrm{a}} \\
(10.21)\end{array}$ & $\begin{array}{c}1.6^{\mathrm{a}} \\
(4.62)\end{array}$ & $\begin{array}{c}68.0^{\mathrm{d}} \\
(55.71)\end{array}$ \\
\hline & Ooty & $\begin{array}{c}84.0 \mathrm{j} \\
(66.76)\end{array}$ & $\begin{array}{c}64.8^{\mathrm{I}} \\
(53.65)\end{array}$ & $\begin{array}{c}28.8^{\mathrm{I}} \\
(32.36)\end{array}$ & $\begin{array}{c}88.0 \mathrm{j} \\
(72.00)\end{array}$ \\
\hline \multirow[t]{4}{*}{ Premier } & Gudiatham & $\begin{array}{c}23.2^{\mathrm{d}} \\
(28.23)\end{array}$ & $\begin{array}{c}4.0^{\mathrm{a}} \\
(10.21)\end{array}$ & $\begin{array}{c}3.2^{\mathrm{b}} \\
(7.90)\end{array}$ & $\begin{array}{c}36.0^{\mathrm{b}} \\
(36.65)\end{array}$ \\
\hline & Hosur & $\begin{array}{c}64.0^{f} \\
(53.19)\end{array}$ & $\begin{array}{c}24.8^{\mathrm{d}} \\
(29.77)\end{array}$ & $\begin{array}{c}7.2^{\mathrm{d}} \\
(15.56)\end{array}$ & $\begin{array}{c}78.0 \mathrm{~g} \\
(62.40)\end{array}$ \\
\hline & Kodaikanal & $\begin{array}{c}68.0 \mathrm{~g} \\
(55.63)\end{array}$ & $\begin{array}{c}36.0^{f} \\
(36.81)\end{array}$ & $\begin{array}{c}14.4^{\mathrm{e}} \\
(22.08)\end{array}$ & $\begin{array}{c}68.0^{\mathrm{d}} \\
(55.71)\end{array}$ \\
\hline & Tirupathur & $\begin{array}{c}10.4^{\mathrm{a}} \\
(18.42)\end{array}$ & $\begin{array}{c}5.6^{\mathrm{b}} \\
(10.31)\end{array}$ & $\begin{array}{c}1.6^{\mathrm{a}} \\
(4.62)\end{array}$ & $\begin{array}{c}68.0^{\mathrm{d}} \\
(55.89)\end{array}$ \\
\hline \multirow[t]{4}{*}{ Rajmash DPU-88-4 } & Melchengam & $\begin{array}{c}18.4^{\mathrm{b}} \\
(25.26)\end{array}$ & $\begin{array}{c}9.6^{c} \\
(17.33)\end{array}$ & $\begin{array}{c}3.2^{\mathrm{b}} \\
(7.90)\end{array}$ & $\begin{array}{c}24.0^{\mathrm{a}} \\
(28.80)\end{array}$ \\
\hline & Nanjanadu & $\begin{array}{c}78.4^{\mathrm{I}} \\
(62.40)\end{array}$ & $\begin{array}{c}62.4^{\mathrm{h}} \\
(53.23)\end{array}$ & $\begin{array}{c}21.6 \mathrm{~g} \\
(27.60)\end{array}$ & $\begin{array}{c}82.0^{\mathrm{h}} \\
(65.36)\end{array}$ \\
\hline & Ooty & $\begin{array}{c}88.0^{k} \\
(73.30)\end{array}$ & $\begin{array}{c}69.6 \mathrm{j} \\
(56.62)\end{array}$ & $\begin{array}{c}24.0^{\mathrm{h}} \\
(29.17)\end{array}$ & $\begin{array}{c}86.0^{\mathrm{I}} \\
(70.67)\end{array}$ \\
\hline & Yercaud & $\begin{array}{c}60.8^{\mathrm{e}} \\
(51.35)\end{array}$ & $\begin{array}{c}45.6^{\mathrm{g}} \\
(42.46)\end{array}$ & $\begin{array}{c}18.4^{f} \\
(24.91)\end{array}$ & $\begin{array}{c}70.0^{\mathrm{e}} \\
57.51)\end{array}$ \\
\hline \multirow[t]{4}{*}{ Watex } & Hosur & $\begin{array}{c}91.2^{1} \\
(73.01)\end{array}$ & $\begin{array}{c}94.4^{1} \\
(78.05)\end{array}$ & $\begin{array}{c}68.0^{\mathrm{k}} \\
(55.65)\end{array}$ & $\begin{array}{c}98.0^{1} \\
(86.31)\end{array}$ \\
\hline & Kodaikanal & $\begin{array}{c}84.8^{\mathrm{j}} \\
(67.14)\end{array}$ & $\begin{array}{c}92.0^{\mathrm{k}} \\
(74.10)\end{array}$ & $\begin{array}{c}63.2^{\mathrm{j}} \\
(52.73)\end{array}$ & $\begin{array}{c}96.0^{\mathrm{k}} \\
(82.63)\end{array}$ \\
\hline & Nanjanadu & $\begin{array}{c}98.4 \mathrm{~m} \\
(85.39)\end{array}$ & $\begin{array}{c}98.4^{\mathrm{n}} \\
(85.39)\end{array}$ & $\begin{array}{l}88.88^{m} \\
(72.63)\end{array}$ & $\begin{array}{l}100.0^{\mathrm{m}} \\
(90.00)\end{array}$ \\
\hline & Ooty & $\begin{array}{c}98.4^{\mathrm{m}} \\
(85.39)\end{array}$ & $\begin{array}{c}96.8^{\mathrm{m}} \\
(82.10)\end{array}$ & $\begin{array}{c}76.8^{1} \\
(61.56)\end{array}$ & $\begin{array}{l}100.0^{\mathrm{m}} \\
(90.00)\end{array}$ \\
\hline
\end{tabular}

\footnotetext{
* Mean of five replications
}

(Figures in paranthesis are arc sine transformed values) In a column, means followed by a common letter are not significantly different at $5 \%$ level by DMRT

The seed samples collected from higher elevation recorded poor per cent seed germination and seedling vigour compared with seed samples collected from plains (Table 2). Skirpta et al. (1986) reported similar reduction in seed germination and inhibition of seedling development of soybean seeds infected with C. truncatum.

Regarding the association of $C$. lindemuthianum with discoloured seeds, the cultivar Watex recorded maximum per cent discoloured seeds with $C$. lindemuthianum. 
Table 2

Effect of $C$. lindemuthianum seed infection on french bean seed germination

\begin{tabular}{|c|c|c|c|c|}
\hline Variety & $\begin{array}{l}\text { Place of } \\
\text { collection }\end{array}$ & $\begin{array}{c}\text { Germination } \\
(\%)\end{array}$ & $\begin{array}{c}\text { Abnormal } \\
\text { seedlings* }(\%)\end{array}$ & $\begin{array}{c}\text { Dead seed* } \\
(\%)\end{array}$ \\
\hline \multirow[t]{4}{*}{ Arkakomal } & Hosur & $\begin{array}{c}49.6^{\mathrm{b}} \\
(44.75)\end{array}$ & $\begin{array}{c}37.6^{b} \\
(37.74)\end{array}$ & $\begin{array}{c}12.8^{\mathrm{a}} \\
(20.65)\end{array}$ \\
\hline & Madurai & $\begin{array}{c}72.0^{\mathrm{a}} \\
(58.17)\end{array}$ & $\begin{array}{c}17.6^{\mathrm{c}} \\
(24.66)\end{array}$ & $\begin{array}{l}10.4 \mathrm{a}^{\mathrm{b}} \\
(16.77)\end{array}$ \\
\hline & Melchengam & $\begin{array}{c}95.2^{\mathrm{a}} \\
(78.81)\end{array}$ & $\begin{array}{c}3.2^{\mathrm{a}} \\
(9.23)\end{array}$ & $\begin{array}{c}1.6^{c} \\
(4.62)\end{array}$ \\
\hline & Ooty & $\begin{array}{c}44.8^{\mathrm{c}} \\
(41.86)\end{array}$ & $\begin{array}{c}48.0^{\mathrm{a}} \\
(43.85)\end{array}$ & $\begin{array}{c}7.2^{\mathrm{ab}} \\
(15.24)\end{array}$ \\
\hline \multirow[t]{4}{*}{ Premier } & Gudiatham & $\begin{array}{c}84.8^{\mathrm{a}} \\
(67.53)\end{array}$ & $\begin{array}{c}12.8^{a} \\
(20.65)\end{array}$ & $\begin{array}{c}2.4^{\mathrm{a}} \\
(5.59)\end{array}$ \\
\hline & Hosur & $\begin{array}{c}73.6^{\mathrm{a}} \\
(59.51)\end{array}$ & $\begin{array}{c}20.0^{\mathrm{a}} \\
(26.12)\end{array}$ & $\begin{array}{c}6.4^{\mathrm{ab}} \\
(12.93)\end{array}$ \\
\hline & Kodaikanal & $\begin{array}{c}73.6^{\mathrm{a}} \\
(59.51)\end{array}$ & $\begin{array}{c}17.6^{\mathrm{a}} \\
(24.31)\end{array}$ & $\begin{array}{c}8.8^{\mathrm{a}} \\
(16.99)\end{array}$ \\
\hline & Tirupathur & $\begin{array}{c}72.8^{a} \\
(59.29)\end{array}$ & $\begin{array}{c}16.0^{\mathrm{a}} \\
(23.16)\end{array}$ & $\begin{array}{c}11.2^{\mathrm{a}} \\
(17.15)\end{array}$ \\
\hline \multirow[t]{4}{*}{ Rajmash DPU-88-4 } & Kelamangalam & $\begin{array}{c}76.8^{\mathrm{a}} \\
(61.38)\end{array}$ & $\begin{array}{c}17.6^{\mathrm{b}} \\
(24.56)\end{array}$ & $\begin{array}{c}5.6^{\mathrm{a}} \\
(13.49)\end{array}$ \\
\hline & Nanjanadu & $\begin{array}{c}56.0^{\mathrm{b}} \\
(48.47)\end{array}$ & $\begin{array}{c}32.8^{\mathrm{a}} \\
(34.80)\end{array}$ & $\begin{array}{c}11.2^{\mathrm{b}} \\
(19.39)\end{array}$ \\
\hline & Ooty & $\begin{array}{c}52.0^{\mathrm{b}} \\
(46.16)\end{array}$ & $\begin{array}{c}35.2^{\mathrm{a}} \\
(36.29)\end{array}$ & $\begin{array}{c}12.0^{\mathrm{b}} \\
(20.06)\end{array}$ \\
\hline & Yercaud & $\begin{array}{c}72.0^{\mathrm{b}} \\
(58.38)\end{array}$ & $\begin{array}{c}20.8^{b} \\
(26.77)\end{array}$ & $\begin{array}{c}7.2^{\mathrm{a}} \\
(15.24)\end{array}$ \\
\hline \multirow[t]{4}{*}{ Watex } & Hosur & $\begin{array}{c}60.0^{\mathrm{a}} \\
(50.81)\end{array}$ & $\begin{array}{c}24.8^{b} \\
(29.68)\end{array}$ & $\begin{array}{c}15.2^{\mathrm{a}} \\
(22.68)\end{array}$ \\
\hline & Kelamangalam & $\begin{array}{c}50.4^{b} \\
(45.24)\end{array}$ & $\begin{array}{c}29.6^{\mathrm{ab}} \\
(32.91)\end{array}$ & $\begin{array}{c}20.0^{\mathrm{a}} \\
(26.37)\end{array}$ \\
\hline & Nanjanadu & $\begin{array}{c}51.2^{\mathrm{b}} \\
(45.69)\end{array}$ & $\begin{array}{c}30.4^{\mathrm{ab}} \\
(33.43)\end{array}$ & $\begin{array}{c}18.4^{\mathrm{a}} \\
(24.81)\end{array}$ \\
\hline & Ooty & $\begin{array}{c}43.2^{\mathrm{b}} \\
(40.93)\end{array}$ & $\begin{array}{c}38.4^{\mathrm{a}} \\
(38.13)\end{array}$ & $\begin{array}{l}18.4^{\mathrm{a}} \\
(24.81)\end{array}$ \\
\hline
\end{tabular}

* Mean of five replications

(Figures in paranthesis are arc sine transformed values) In a column, means followed by a common letter are not significantly different at $5 \%$ level by DMRT

The infected seeds showed characteristic brown spots with whitish center surrounded by a pale brown to dark brown margin. Arkakomal recorded lowest seed infection (Table 3). The results indicated that there was direct correlation exist between discoloured seeds and seed infection. Similar observations on the association of $C$. lindemuthianum in discoloured seeds of french bean have been reported by several workers (Rodriguez, 1984; Goulart, 1988; Tylkowska and Dorna, 1990). 
Table 3

Association of $C$. lindemuthianum with discoloured french bean seeds

\begin{tabular}{lccc}
\hline Variety & No. of seeds tested & $\begin{array}{c}\text { Discoloured } \\
\text { seeds* }(\%)\end{array}$ & $\begin{array}{c}\text { C. lindemuthianum } \\
\text { associated with } \\
\text { discoloured } \\
\text { seeds* }(\%)\end{array}$ \\
\hline Arkakomal & 400 & $37.25^{\mathrm{c}}$ & $20.50^{\mathrm{d}}$ \\
& & $(37.61)$ & $(26.91)$ \\
Premier & 400 & $58.75^{\mathrm{b}}$ & $36.50^{\mathrm{c}}$ \\
& & $(50.04)$ & $(37.17)$ \\
Rajmash DPU-88-4 & 400 & $60.50^{\mathrm{b}}$ & $40.75^{\mathrm{b}}$ \\
Watex & 400 & $(51.06)$ & $(39.67)$ \\
& & $67.50^{\mathrm{a}}$ & $47.25^{\mathrm{a}}$ \\
& & $(55.25)$ & $(43.42)$ \\
\hline
\end{tabular}

* Mean of five replications

(Figures in paranthesis are arc sine transformed values) In a column, means followed by a common letter are not significantly different at $5 \%$ level by DMRT

The results from the effect of $C$. lindemuthianum seed infection on seed quality revealed that there was a significant reduction in seed size and weight in all four varieties. Among the four varieties, Watex recorded the maximum per cent seed infection and reduction in seed size and weight, while Arkakomal recorded minimum seed infection with least reduction in seed size and weight (Table 4). The loss in seed weight and shriveling of seeds due to fungal infection was reported by Gaikward et al. (1993) in soybean and by Subramanian (1988) in rice. It was observed that upon the extent of seed infection there was a reduction in length and breadth of seeds.

\section{Table 4}

Effect of C. lindemuthianum seed infection on seed size and weight

\begin{tabular}{|c|c|c|c|c|c|c|c|c|c|c|}
\hline \multirow[b]{2}{*}{ Variety } & \multirow[b]{2}{*}{$\begin{array}{c}\text { Seed } \\
\text { infection } \\
(\%)\end{array}$} & \multicolumn{3}{|c|}{ Length $(\mathrm{mm})^{*}$} & \multicolumn{3}{|c|}{ Breadth $(\mathrm{mm})^{*}$} & \multicolumn{3}{|c|}{100 Seed weight $(\mathrm{g})^{*}$} \\
\hline & & Healthy & Infected & $\begin{array}{c}\text { Reduction } \\
\text { over healthy } \\
\text { seed }\end{array}$ & Healthy & Infected & $\begin{array}{c}\text { Reduction } \\
\text { over healthy } \\
\text { seed }\end{array}$ & Healthy & Infected & $\begin{array}{c}\text { Reduction } \\
\text { over healthy } \\
\text { seed }\end{array}$ \\
\hline Arkakomal & $\begin{array}{c}57.0^{\mathrm{a}} \\
(49.02)\end{array}$ & $13.41^{\mathrm{c}}$ & $11.39 \mathrm{c}$ & 15.06 & $7.75^{\mathrm{a}}$ & $6.64^{*}$ & 14.32 & $34.94^{*}$ & $33.07^{*}$ & 5.35 \\
\hline Premier & $\begin{array}{c}59.0^{\mathrm{ab}} \\
(50.20)\end{array}$ & $14.63^{\mathrm{b}}$ & $12.15^{\mathrm{b}}$ & 16.95 & 7.34 & $6.11^{*}$ & 16.76 & $36.22^{*}$ & $33.75^{*}$ & 6.82 \\
\hline $\begin{array}{l}\text { Rajmash } \\
\text { DPU-88-4 }\end{array}$ & $\begin{array}{c}66.0^{\mathrm{ab}} \\
(54.33)\end{array}$ & $15.48^{\mathrm{a}}$ & $12.81^{\mathrm{b}}$ & 17.25 & 6.55 & $5.60^{*}$ & 14.50 & $38.29^{*}$ & $34.51^{*}$ & 9.87 \\
\hline Watex & $\begin{array}{c}70.0^{\mathrm{a}} \\
(56.80)\end{array}$ & $12.61^{\mathrm{d}}$ & $10.11^{\mathrm{a}}$ & 19.83 & 5.75 & $4.27^{*}$ & 25.74 & $31.40^{*}$ & $26.58^{*}$ & 15.35 \\
\hline
\end{tabular}

* Mean of five replications

(Figures in paranthesis are arc sine transformed values) In a column, means followed by a common letter are not significantly different at $5 \%$ level by DMRT 
In the present study, seed coat and cotyledon showed maximum infection of $C$. lindemuthianum, while the embryonic axis showed very low infection. The seed samples of all the four varieties recorded maximum infection of $C$. lindemuthianum on seed coat. The variety Watex had higher seed infection leads to higher seed coat and cotyledonary infection and low infection of embryonic axis (Table 5). Franca Neti and West (1989) also made similar observation in soybean that the $C$. truncatum infection was mainly restricted to seed coats.

\section{Table 5}

C. lindemuthianum infection in seed and seed parts

\begin{tabular}{lcccc}
\hline Variety & $\begin{array}{c}\text { Seed } \\
\text { infection }(\%)^{*}\end{array}$ & $\begin{array}{c}\text { Seed coat } \\
\text { infection }(\%)^{*}\end{array}$ & $\begin{array}{c}\text { Cotyledonary } \\
\text { infection }(\%)^{*}\end{array}$ & $\begin{array}{c}\text { Embryonic axis } \\
\text { infection }(\%)^{*}\end{array}$ \\
\hline Arkakomal & 57.00 & $75.2^{\mathrm{bc}}$ & $66.4^{\mathrm{a}}$ & $11.2^{\mathrm{b}}$ \\
Premier & 59.00 & $(60.35)$ & $(54.83)$ & $(19.08)$ \\
& & $70.4^{\mathrm{c}}$ & $54.4^{\mathrm{b}}$ & $10.4^{\mathrm{b}}$ \\
Rajmash DPU-88-4 & 66.00 & $(57.21)$ & $(47.54)$ & $(18.63)$ \\
Watex & 70.00 & $80.0^{\mathrm{ab}}$ & $60.8^{\mathrm{ab}}$ & $8.0^{\mathrm{b}}$ \\
& & $(63.66)$ & $(51.25)$ & $(16.22)$ \\
& & $86.4^{\mathrm{a}}$ & $67.2^{\mathrm{a}}$ & $19.2^{\mathrm{a}}$ \\
& & $(68.58)$ & $(55.11)$ & $(25.78)$ \\
\hline
\end{tabular}

* Mean of five replications

(Figures in paranthesis are arc sine transformed values) In a column, means followed by a common letter are not significantly different at $5 \%$ level by DMRT

The result on the susceptible age at which the anthracnose incidence occurs in a severe form revealed that the susceptibility increased with the increase in age of plants and later it declined. The plants received inoculum on 45th day recorded the maximum disease incidence with maximum resultant seed infection and minimum seed yield/plant and 100 seed weight (Table 6). This was in agreement with the earlier findings of Sindhan and Bose (1981). They observed that the plants at all ages were susceptible to the disease, but the young plants were less susceptible in comparison to older plants and the lower leaves were more vulnerable to infection than younger ones. Higher susceptibility of the older plants may be due to the decrease in total sugar and phenolic content as observed in brown sarcon by Chahal (1986).

With this it is concluded that the results emerged from the above investigation have brought out the following points:

(i) Agar plate method by using potato dextrose agar was found to be an ideal technique to detect $C$. lindemuthianum from french bean.

(ii) The seeds collected from temperate regions were found to record more seed borne infection than tropical regions.

(iii) A direct correlation was observed between per cent discoloured seed and seed infection which caused lesser seed weight. 


\section{Table 6}

Effect of plant age on disease incidence and seed transmission of $C$. lindemuthianum

\begin{tabular}{|c|c|c|c|c|c|}
\hline Plant age & $\begin{array}{c}\text { Disease } \\
\text { incidence }(\%)^{*}\end{array}$ & $\begin{array}{c}\text { Pod } \\
\text { infection }(\%)^{*}\end{array}$ & $\begin{array}{l}\text { Resultant seed } \\
\text { infection }(\%)^{*}\end{array}$ & $\begin{array}{c}\text { Seed } \\
\text { yield/plant }(g)^{*}\end{array}$ & $\begin{array}{c}100 \text { Seed } \\
\text { weight }(g)^{*}\end{array}$ \\
\hline 15 & $\begin{array}{c}10.15^{\mathrm{d}} \\
(18.58)\end{array}$ & $\begin{array}{l}17.27^{\mathrm{c}} \\
(24.37)\end{array}$ & $\begin{array}{c}7.87 \mathrm{~d} \\
(16.18)\end{array}$ & $15.17^{\mathrm{a}}$ & $30.83^{a}$ \\
\hline 30 & $\begin{array}{l}47.40^{c} \\
(43.50)\end{array}$ & $\begin{array}{l}36.31^{\mathrm{b}} \\
(37.01)\end{array}$ & $\begin{array}{l}19.66^{c} \\
(26.28)\end{array}$ & $13.93^{\mathrm{b}}$ & $28.95^{\mathrm{b}}$ \\
\hline 45 & $\begin{array}{r}70.77 \mathrm{a} \\
(57.27)\end{array}$ & $\begin{array}{r}66.87 \mathrm{a} \\
(54.88)\end{array}$ & $\begin{array}{l}56.75^{\mathrm{a}} \\
(48.91)\end{array}$ & $8.83^{\mathrm{d}}$ & $24.29 \mathrm{~d}$ \\
\hline 60 & $\begin{array}{l}65.13^{\mathrm{b}} \\
(59.79)\end{array}$ & $\begin{array}{l}59.70^{\mathrm{a}} \\
(56.21)\end{array}$ & $\begin{array}{l}48.77^{b} \\
(44.29)\end{array}$ & $9.80^{\mathrm{c}}$ & $25.10^{c}$ \\
\hline
\end{tabular}

* Mean of five replications

(Figures in paranthesis are arc sine transformed values) In a column, means followed by a common letter are not significantly different at $5 \%$ level by DMRT

(iv) Pathogen was found to be associated mostly in seed coat and cotyledons.

(v) The 45-day-old plants were highly susceptible to the infection and lead to maximum disease incidence and resultant seed infection.

\section{Literature}

Ansleme, C. and Champion, R. (1981): Bean anthracnose. ISTA Handbook on seed health testing. International Seed Testing Association. Working sheet. No. 45.

Arya, C. M., Dhengra, O. D. and Kushalappa, A. C. (1986): Incidence of anthracnose in bean under different inoculum pressures. Fitopathologia Brasileria 11, 795-801.

Chahal, A. S. (1986): Relationship of Alternaria blight with the age of brown sarcon. Indian J. Mycol. Pl. Pathol. 10, 166-167.

Davis, J. (1988): New bean varieties for Burundi. Hojas de Frijol. 10, 4-5.

Franca Neti, J. B. and West, S. H. (1989): Effects of Colletotrichum truncatum and Cercospora kikuchi on viability and quality of seed born seeds. J. Seed Tech. 13, 136-149.

Gaikward, S. J., Gohokar, R. T. and Meshram, S. V. (1993): Occurrence of pod blight of soyabean caused by Colletotrichum dematium. Maharastra Agrl. Univ. 18, 489-491.

Goulart, A. C. P. (1988): Bean diseases in the northen region of the state of Minas Gerais. Fitopathologia Brasileria 13, 230-232.

ISTA (1985): International rules for seed Testing. Seed Sci. and Technol. 13, 484-487.

Kunwar, J. K., Singh, T., Machado, C. C. and Sinclair, J. B. (1985): Histopathology of soyabean and seedling infection by Macrophomina phaseolina. Phytopathology 76, 532-535.

Limonard, T. (1966): A modified blotter test for seed health. Neth. J. Pl. Path. 72, 319-321.

Mew, T. W. and Misra, J. K. (1994): A manual of rice seed health testing. IRRI, Philippines.

Neergaard, Paul and Saab, Adib (1962): Seed health testing of rice. A contribution to development of laboratory testing methods. Indian Phytopath. 15, 85-111.

Rodriguez, E. N. (1984): Identification, incidence, transmission and control of seed borne disease of some Philippine legumes. M. Sc Thesis, college Laguna, University of Philippines. 
Sindhan, G. S. and Bose, S. K. (1981): Epidemiology of anthracnose of french bean caused by Colletotrichum lindemuthianum. Indian Phytopath. 34, 484-487.

Skirpta, O. V., Sizova, T. P. and Babeva, E. N. (1986): Fungi on soyabean seeds in Abkhazia. Mikologiya I Fitopatologiya, Moscow, 20, 306-308.

Subramanian, K. S. (1988): Studies on grain discolouration of rice caused by Drechsclera oryzae (Breda. De. Haan) subram and Jain and Curvularia lunta (Wakker) Boedijn. M. Sc(Ag) Thesis, Tamilnadu Agricultural University, Coimbatore p. 208.

Tylkowska, K. and Dorna, H. (1990): The usefulness of two methods for detecting pathogenic fungi in common bean seeds. Hodowla Roslin. Aklimatyzacja I Nasiennictwo 32, 101-110.

Zaumeyer, W. J. and Thomas, H. R. (1957): A monographic study of bean diseases and methods of their control. USDA Tech Bull. 868 (Revised ed.) 255p. 